\title{
Concise Commentary: III Preparation for Managing Liver Illness Among Incoming Medical Residents
}

\author{
Deborah D. Proctor ${ }^{1}$
}

Accepted: 16 July 2021 / Published online: 7 August 2021

( ) The Author(s), under exclusive licence to Springer Science+Business Media, LLC, part of Springer Nature 2021

Chronic liver disease, which is rising in prevalence in the US, is increasingly evaluated and managed by general internists and hospitalists $[1,2]$. There is no question that the pathophysiology of the disease itself, its diagnosis, and management of its complications and sequelae need to be taught and understood by all medical students.

To understand the level of knowledge regarding chronic liver disease (CLD) among medical students, Dr. Pan et al., in this issue of Digestive Diseases and Sciences, (3) distributed an anonymous survey to two consecutive years of incoming internal medicine residents at the University of Chicago in order to assess their comfort level and knowledge of CLD. Their responses indicated that although almost all (98\%) believed that hepatology training was important, fewer than a quarter (22\%) acknowledged that they had taken a GI or hepatology rotation during medical school and fewer than half (42\%) reported that their hepatology education was sufficient. Two thirds (67\%) noted that they were comfortable interpreting liver function tests, but the self-reported comfort level for managing a cirrhotic patient, performing a paracentesis, or managing a patient post-liver transplant was adequate in less than one-third of respondents. The incoming interns rated their comfort level far lower for managing patients with liver disease than for patients with congestive heart failure or COPD.

This study highlights the deficiency in medical education regarding teaching and exposure to patients with CLD. As the prevalence of CLD has risen, teaching the evaluation and management of this important disease has lagged behind other topics in medical school curricula. Although these were incoming interns in a single internal medicine residency program, the survey respondents represented 30 different medical schools suggesting that this issue is

Deborah D. Proctor

Deborah.proctor@yale.edu

1 Yale University School of Medicine, 40 Temple Street, Suite 1A, New Haven, CT 06510, USA widespread. As the authors point out, a forthcoming national survey with a broader group of graduates will provide additional information on which to base curricular changes.

As the prevalence of CLD rises, the number of hepatologists needed to manage these complex patients has correspondingly risen. The authors point out that developing a sufficient comfort level with CLD early in training might influence a resident's decision to pursue hepatology as a field of practice. Moreover, as complex CLD patients are increasingly being managed by non-hepatologists, general internists and hospitalists will need to acquire an adequate knowledge base. Therefore, there are multiple reasons to graduate from medical school with a sufficient understanding of the pathophysiology of, and experience with, chronic liver disease in order to enable the competent management of these complex patients. This current study begins to address the knowledge gap in training as it pertains to hepatology.

\section{Declarations}

Conflict of interest The author declares no conflict of interest.

\section{References}

1. https://www.cdc.gov/nchs/fastats/liver-disease.htm. Accessed June 18,2021

2. Hirode G, Saab S, Wong RJ. Trends in the burden of chronic liver disease among hospitalized US adults. JAMA Netw Open. 2020;3:e201997. https://doi.org/10.1001/jamanetworkopen.2020.1997.

3. Pan AY, Zilberstein NF, Faman JM, McConville JF, Mikolajczyk AE. Recently graduated medical students lack exposure to and comfort with chronic liver disease. Dig Dis Sci. (Epub ahead of print). https://doi.org/10.1007/s10620-021-07182-0.

Publisher's Note Springer Nature remains neutral with regard to jurisdictional claims in published maps and institutional affiliations. 